\title{
The Effect of HARQ Procedure on the Performance of LTE System
}

\section{Safwan H. Fasola}

Assistant Lecturer. Elect.Eng. College/ Comm. Dept.

\author{
Firas S. Al-Sharbaty
}

Assistant Lecturer.

Eng. College/ Elect.Dept.

\begin{abstract}
In this paper, the parameters of the LTE system in DL are investigated. These parameters include the effect of the Hybrid Adaptive Repeat and Request henceforth (HARQ) on the Signal to Noise Ratio (SNR), Block Error Rate (BLER), and throughput. The paper deals with three cases of Channel Quality Indicator (CQI): 3,7 and 15. The results showed that the HARQ procedure can improve the BLER and the throughput when the retransmission between the evolved base station (eNodeB) and a User Equipment (UE) increased.
\end{abstract}

Keyword:-Channel Quality Indicator (CQI), Hybrid Adaptive Repeat and Request (HARQ), Long Term Evolution (LTE), User Equipment (UE).

$$
\begin{aligned}
& \text { تاثير اجرائية طلب الاعادة المتكيف الهجين على نظام امد التطور الطويل } \\
& \text { فراس سامي الشربتي } \\
& \text { مدرس مساعد المسير } \\
& \text { صفوان حفيظ فصولة } \\
& \text { مدرس مساعد } \\
& \text { كلية الزهدسة/قسم الكهرباء ملعاء } \\
& \text { هندسة الاكترونيات/قسم الاتصالات }
\end{aligned}
$$

\section{اخلاصة}

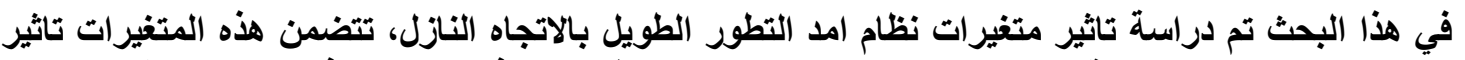

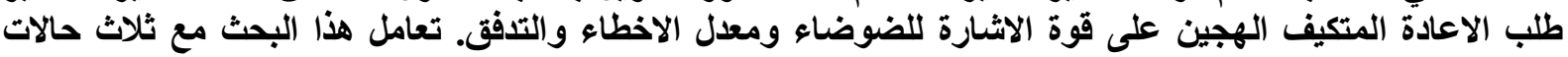

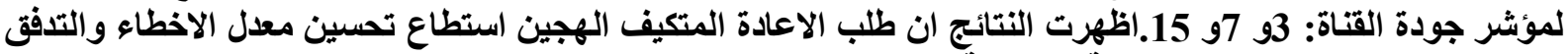

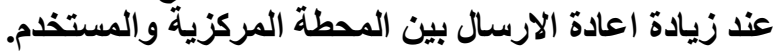

الكلمات المفتاحية:مؤشر جودة القناة، طلب الاعادة المتكيف الهجين، أمد التطور الطويل، المستخدم. 


\section{Introduction:-}

Initially, LTE was begun to ensure the competitiveness of Universal Mobile Telecommunication System(UMTS) where LTE has many features like higher capacity and low delay compared with old systems.In order to fulfill these requirements, LTE is based on OFDMA in the DL direction and SC-FDMA in the UL direction. The idea of OFDMA technique converts the wide band frequency channel carrier into a set of many narrow mutually orthogonal sub-carriers, as shown in Figure (1). The minimum transmission bandwidth is $180 \mathrm{KHz}$ so each user allocated different bandwidth using different number of sub-carriers. The most benefits for using OFDMA multiple access schemes in the DL direction are larger bandwidth and bandwidth flexibility. On the other hand, the benefit of using SC-FDMA in the UL direction is better power amplifier efficiency for the UE[1][2].

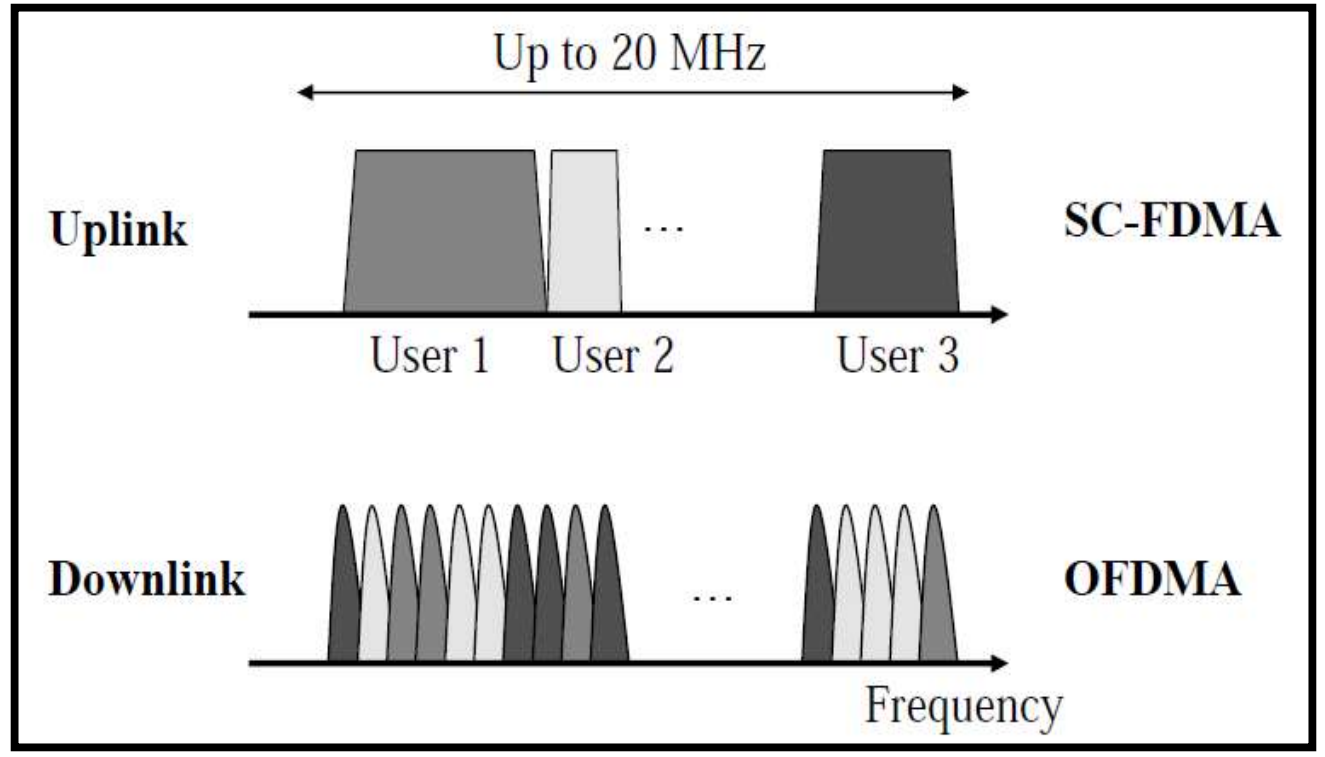

Figure (1): LTE multiple access schemes [2].

The high data rate is one of the key features of the LTE. Therefore, it is needed to use efficient techniques to providing efficiency and reliability of data transmission over a noisy channel. HARQ offers an attractive solution to compensate the error rate by using a combination of Forward Error Correction (FEC) and Automatic Retransmission request.

K. Chung, A. Doufexi and Si. Armour[6]dealt with HARQ. They evaluated the performance of the LTE system after employed the HARQ with different schemes. The difference among these schemes of HARQ depends on the memory requirements, the resources consumption and the delay. M. L. Kawser et.al [7] presented HARQ as a solution to support the performance of LTE system for the weak received signal only. Z. He and F. Zhao[8] intended to maximize the throughput of LTE system using many algorithm of HARQ procedure for many types of modulation and coding schemes.

In this paper, the effect of HARQ on the performance of LTE system is studied. It deals with three cases of channel qualities for UE: worst case, middle case and perfect case. It aims to reach to optimum number of retransmission for each case of channel qualities. 


\section{HARQ in LTE System:-}

\subsection{Backround}

The LTE protocol model specifies the protocols terminated between User Equipment (UE) and eNodeB[6], This protocol stack defines three layers (from down to up): layer one, layer two and layer three[7], as shown in Figure (2). The function of HARQ in the standard of LTE is submitted by the layer two with other functions like segmentation and scheduling. This layer is divided into three sub-layers as shown in Figure (2).Layer one (physical layer) provides the bits transmission. Finally, layer three deals with resources control and application layer[7][8].

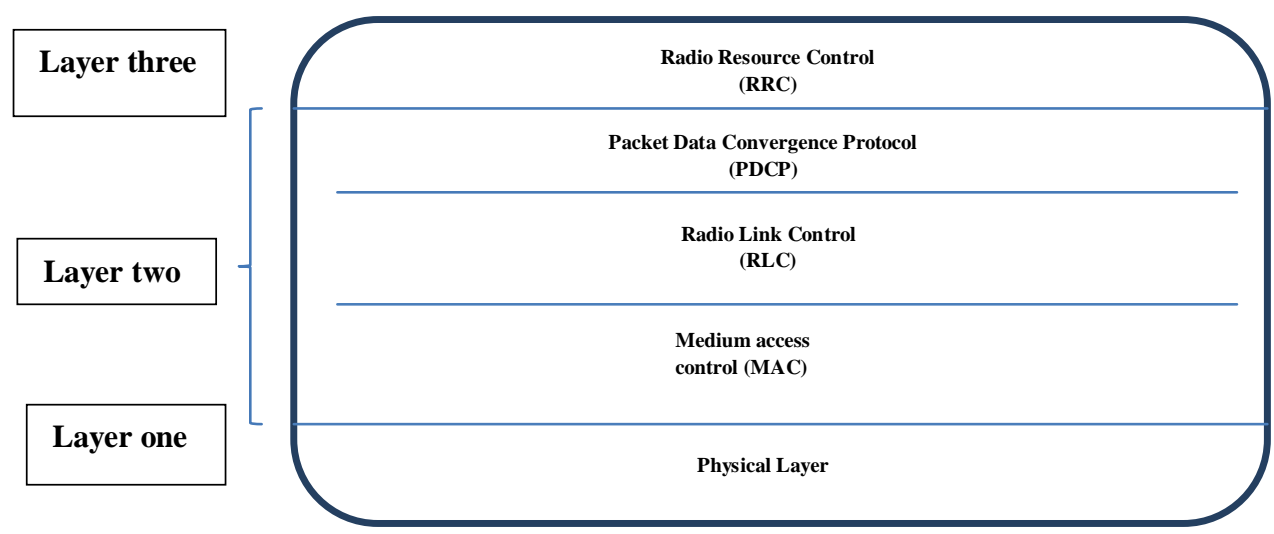

Figure (2): Protocol stack of LTE.

\subsection{Resource block of LTE}

The major concept of resources allocation in LTE system is shown in Figure(3).The spacing between two sub-carriers is typically $15 \mathrm{kHz}$ and each 12 subcarriers with 6 or 7 OFDM symbols (depending on the size of cyclic prefix) called Resource Block (RB). The RB consists of resource elements (REs) group.Each RE represents the basic unit of the resources. The bandwidth for each $\mathrm{RB}$ is $180 \mathrm{kHz}(12 * 15 \mathrm{kHz}=180 \mathrm{KHz})$, so the minimum transmission bandwidth is $180 \mathrm{KHz}$ (one $\mathrm{RB}$ ).Each user allocated different bandwidth using different numbers of RB.

The REs in DL direction are divided into two kinds, REs for data and REs for control[9]. The REs which are devoted to data are carried by physical downlink shared channel (PDSCH). PDSCH can be modulated depends on channel quality of UE which is used QPSK, 16QAM or 64QAM modulations depending on the channel quality of UE.

On the other hand, REs used for control purpose are carried by many channels and physical signals [10]. Physical Hybrid ARQ Indicator Channel (PHICH) is one of the control channels, which deals with the HARQ procedure acknowledgement / negative acknowledgement (ACK/NAKs). ACKs and NAKs confirm the delivery of data or request the retransmission of data blocks received incorrectly[6]. 


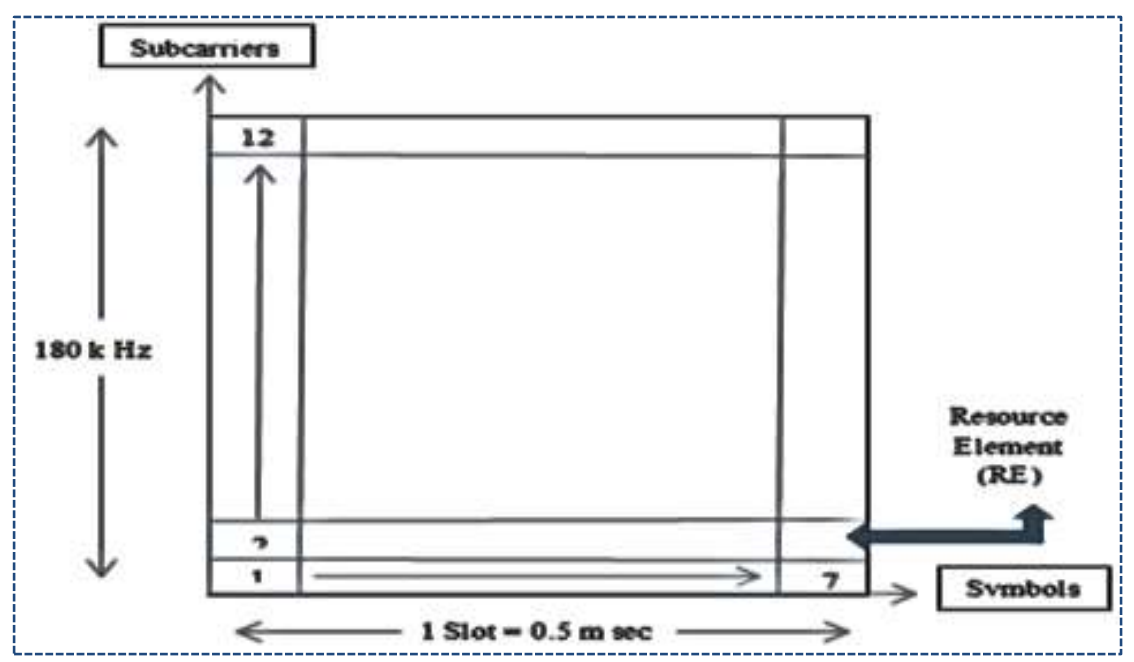

Figure (3): Resource block of LTE.

\subsection{Frame structure of LTE}

The frame structure of LTE consists of ten sub frames, each sub frame contains two slots where the length of one-slot equals to $0.5 \mathrm{~ms}$, see Figure (4).

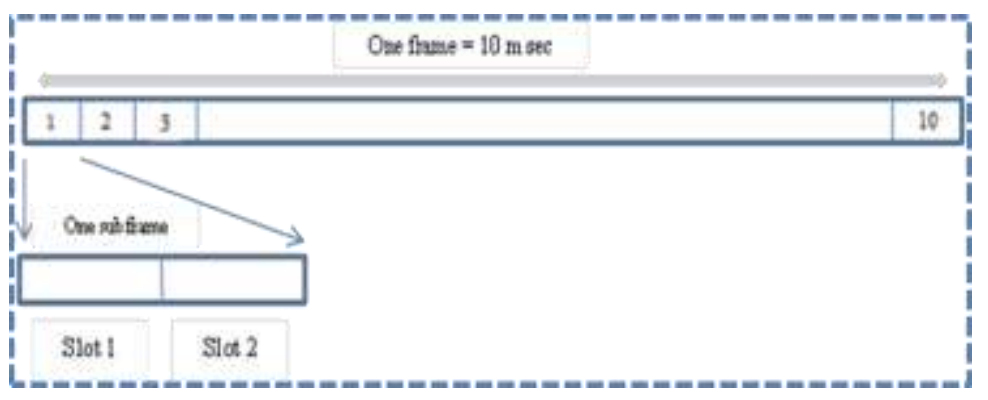

Figure (4): Frame structure of LTE.

The LTE supports two types of frame structures:

i- Type 1 Frame:- Frame is devoted to frequency division duplexing (FDD), it is separated UL on DL.

ii- Type 2 Frame:- Frame is devoted to time division duplexing (TDD), Type 2 is dealt with UL and DL together.

\subsection{Channel Quality Indicator (CQI):-}

The channel quality indicator is a technique which provides the eNodeB information about the link adaption parameters and the data rate that can be received successfully by the UE. The parameters reported by a UE to the eNodeB are signal strength quality, transmission mode, number of antennas and interference situation experienced at the connection time. These parameters will explain the state of UE in enodeB. The value of the CQI which is received at the eNodeB indicates which type of the modulation and coding schemes will be used for the DL direction, see Table 1[2][10]. 


\subsection{Hybrid Adaptive Repeat and Request} (HARQ) procedure:-

HARQ is a technique used in the LTE to reduce the effect of multipath fading channel.It has the ability to enhance SNR and the throughput performance by reducing the error [9]. The HRAQ based on stop and wait procedure. At the beginning the UE decoded the packet which is transmitted from the eNodeB and send the ACK. For NACK, the eNodeB will send a retransmission. The UE will combine the retransmission with the original transmission and will decode again. For successful decoding the UE will send positive acknowledgement (ACK) to the eNode, then the eNodeB will send a new packet for that HARQ process.

It is worth to mention that due to the stop-andwait way of operating, one needs to have multiple HARQ procedure to enable a continuous data flow. In
Table (1): Relationship between CQI and Modulation type [10].

\begin{tabular}{|c|c|c|}
\hline CQI & Modulation & $\begin{array}{l}\text { Coding } \\
\text { Rate }\end{array}$ \\
\hline 0 & - & - \\
\hline 1 & QPSK & $78 / 1024$ \\
\hline 2 & QPSK & $120 / 1024$ \\
\hline 3 & QPSK & $193 / 1024$ \\
\hline 4 & QPSK & $308 / 1024$ \\
\hline 5 & QPSK & $449 / 1024$ \\
\hline 6 & QPSK & $602 / 1024$ \\
\hline 7 & 16QAM & $307 / 1024$ \\
\hline 8 & 16QAM & $490 / 1024$ \\
\hline 9 & 16QAM & $616 / 1024$ \\
\hline 10 & 64QAM & $466 / 1024$ \\
\hline 11 & 64QAM & $567 / 1024$ \\
\hline 12 & 64QAM & $666 / 1024$ \\
\hline 13 & 64QAM & $772 / 1024$ \\
\hline 14 & 64QAM & $873 / 1024$ \\
\hline 15 & 64QAM & $948 / 1024$ \\
\hline
\end{tabular}
LTE the number of processes is fixed to 8 procedures in downlink direction.

The minimum delay of the retransmission is $7 \mathrm{~ms}$ [2][10]. The SNR gains which is provided by using HARQ procedure is: [11]

$S N R_{(i)}=S N R+S N R_{(\text {gain })}$

where $i$ represents the $i t h$ retransmission.

Figure (5) illustrates the procedure of HARQ in case of a single user continuous transmission.

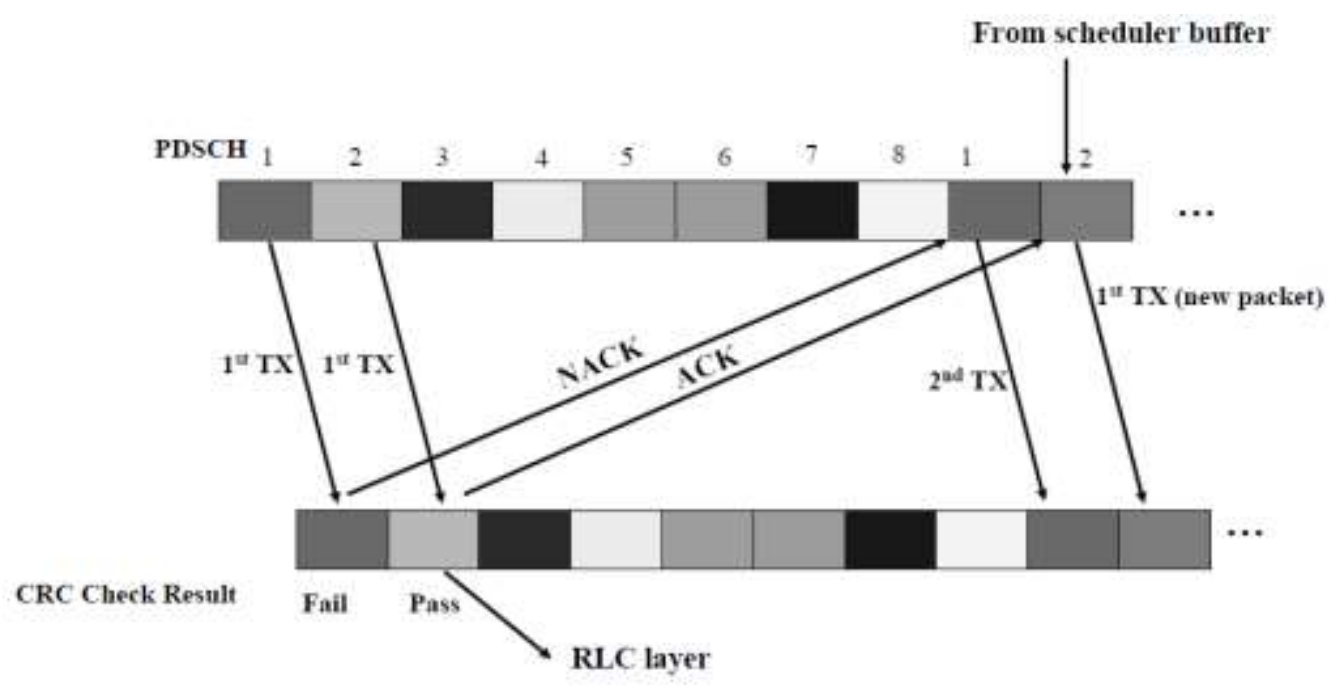

Figure (5): HARQ processes for single UE[10]. 


\section{System Simulation and Evaluation:-}

The simulation under consideration of LTE system is built on the following assumptions:

a) Single Cell - Single User: - covered the communication between one cell and one user only.

b) The cell is divided into three regions as shown in Figure (6). Region 1 represents the worst case (i.e.UE at the edge of the cell), region 2 represents the normal case (i.e.UE in the middle distance between eNodeB and the edge of the cell) and region 3 represents the perfect case (i.e.UE is near from eNodeB).

c) Table (2) demonstrates modulations and coding schemes which are used by UE depending on the CQI.

d) The type of frame is type 1 (FDD).

e) Number of retransmissions (HARQ) is 0, 1, 2 or 3 for each mentioned case.

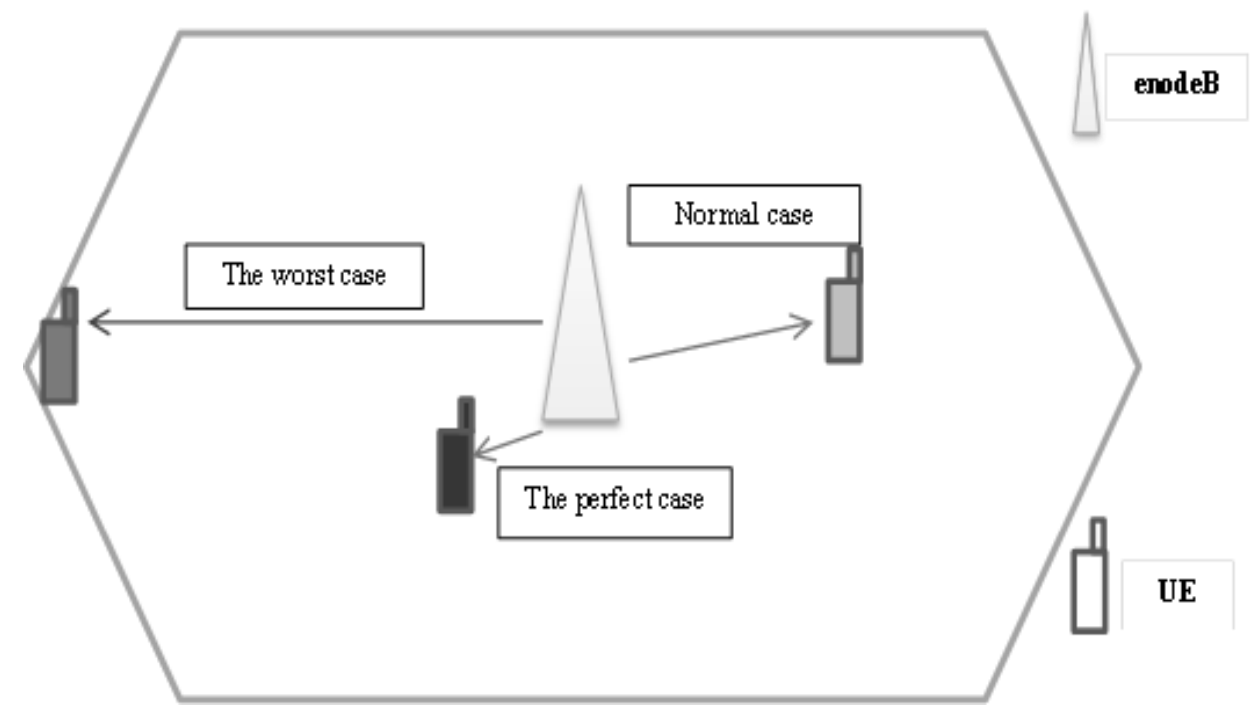

Figure (6): Positions of UE about eNodeB.

Table (2): Modulation and Coding Schemes.

\begin{tabular}{|c|c|c|c|}
\hline CQI & Modulation type & Coding Rate & The Case \\
\hline 3 & QPSK & $193 / 1024$ & UE at the edge of the cell \\
\hline 7 & 16QAM & $307 / 1024$ & $\begin{array}{c}\text { UE at the middle distance between } \\
\text { eNodeB and the border of the cell }\end{array}$ \\
\hline 15 & 64QAM & $948 / 1024$ & UE is near from eNodeB \\
\hline
\end{tabular}

f- The bandwidth equals to $1.4 \mathrm{MHz}$.

g- The details of model assumptions are shown in Table (3) . 
Fasola: The Effect of HARQ Procedure on the Performance of LTE System

Table (3): The details of simulation assumptions.

\begin{tabular}{|c|c|}
\hline Parameter & Value \\
\hline Bandwidth( MHz) & 1.4 \\
\hline The type of frame & Type 1 frame \\
\hline Number of frames & 5000 \\
\hline Types of CQI & $3,7,15$ \\
\hline Number of retransmissions & $0,1,2$ and 3 \\
\hline cyclic prefix & Normal \\
\hline Transmitter and receiver antenna & SISO \\
\hline Coding rate & As shown in table 1 \\
\hline Modulation types & QPSK, 16QAM and 64QAM \\
\hline
\end{tabular}

The link level simulator (v1.0 r-400) based onMATLAB code which is used in this paper is presented by the Institute of Communication and Radio-Frequency Engineering in Vienna University of Technology.The simulator structure consists of three main parts:transmitter, channel model and receiver. The transmitter and receiver can deal with one or more than one antenna. The simulator can deal with many types of channel models such that PedB, PedA, VechB, VechA and others [1].

\section{Results and Discussions:-}

Figure (7) illustrates the relationship between the SNR and BLER for different HARQ retransmissions at $\mathrm{CQI}=3$ which represents the worst case. Using the HARQ retransmissions procedure, SNR is decreased about $1.5 \mathrm{~dB}$ to obtain the same BLER in the case of no HARQ retransmissions as shown in Figure (7). The optimum value of the HARQ is 1-retransmission when the UE at the edge of the cell. The increment of HARQ retransmissions more than one will not enhance the SNR because the link between the eNodeB and UE has higher attenuation and noise due to the distance between them.

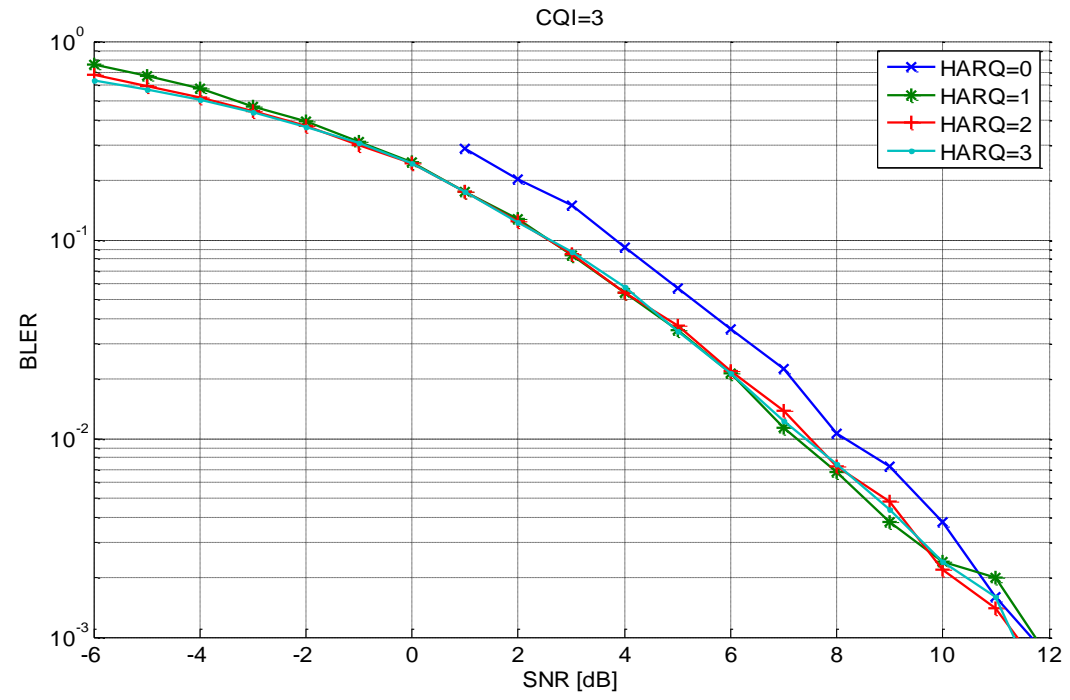

Figure (7): SNR versus BLER when CQI=3. 
The relationship between SNR and throughput for different HARQ retransmissions at $\mathrm{CQI}=3$ is demonstrated in Figure (8). The number of HARQ retransmissions procedure more than one does not improve the throughput. This is related to the type scheme of modulation where QPSK modulation can carry 2-bits only in one symbol, therefore, the gain which is added by HARQ will not improve the throughput. As shown in the Figure for higher SNR (more than $10 \mathrm{~dB}$ ) there is no need to use HARQ procedure.

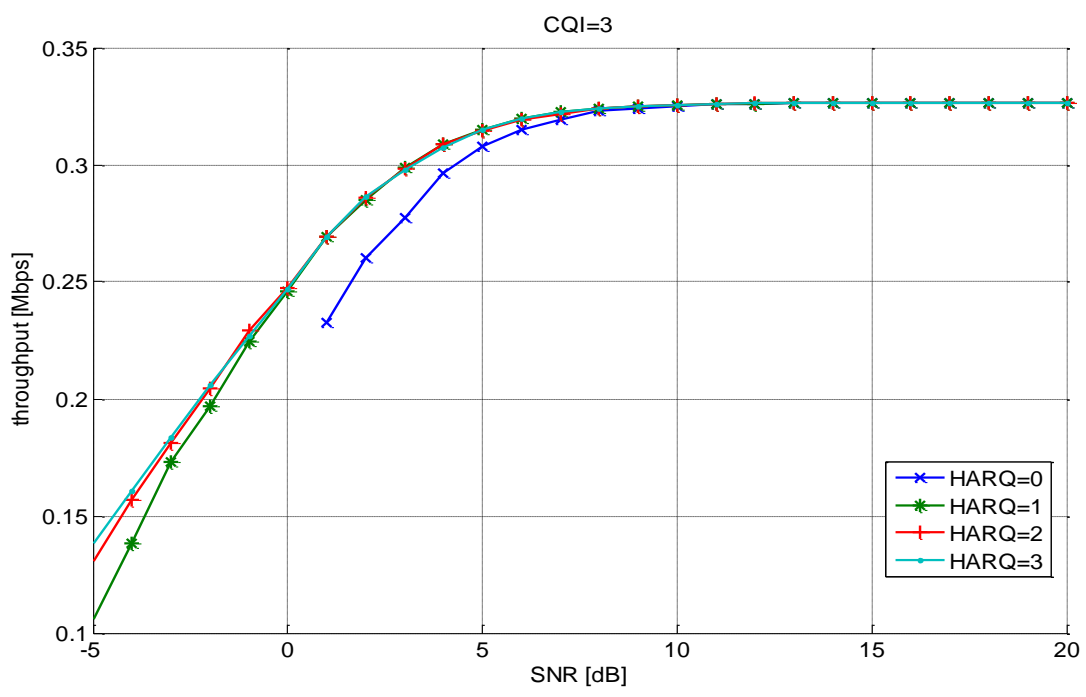

Figure (8): $\mathrm{SNR}$ versus Throughput when $\mathrm{CQI}=3$.

For the normal case of $\mathrm{CQI}=7$,the relationship between the SNR and BLER for different retransmissions HRAQ illustrated in Figure (9). When HARQ procedure is used, SNR is decreased about $2 \mathrm{~dB}$ to obtain the same BLER without using HARQ procedure. When the CQI $=7$, the UE need more SNR compared with CQI $=3$ because of higher modulation scheme (16QAM) is used.

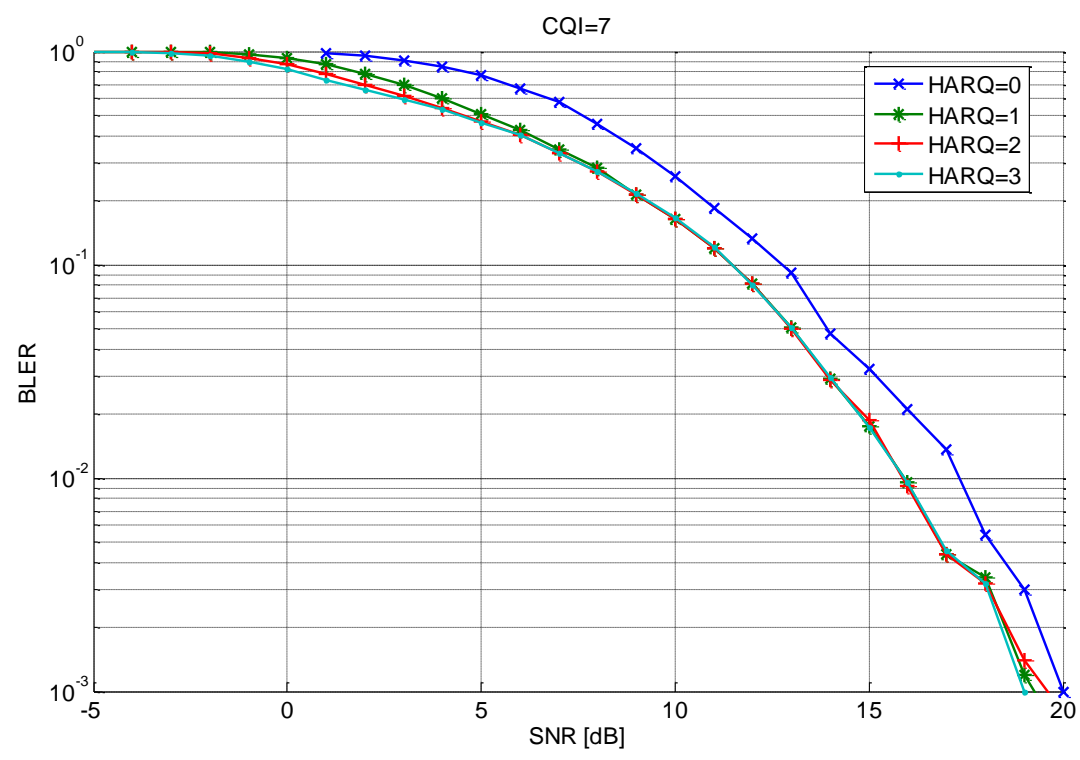

Figure (9): SNR versus BLER when CQI=7. 
Figure (10) illustrates the relationship between SNR and throughput for differnt HARQ retransmissionsat $\mathrm{CQI}=7$. When using HRAQ procedure the throughput is increased about $50 \%$ compared to without using HARQ retransmissions for the same SNR. For higher SNR (more than 16dB) the HARQ procedure does not impact on the throughput and SNR as shown in the figure below:

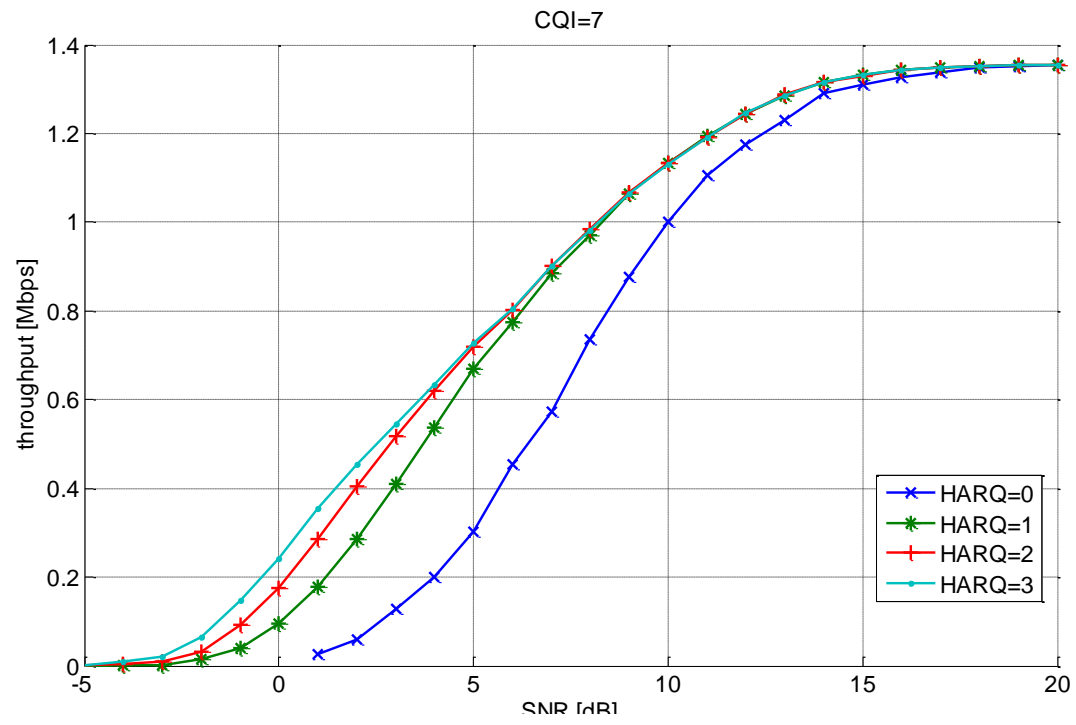

Figure (10): SNR versus Throughput when CQI=7.

Figure (11) demonstrates the relationship between the SNR and BLER for different HARQ retransmissionsat $\mathrm{CQI}=15$ which represents the perfect case. For this case, the UE needs higher $\mathrm{SNR}$ compared to $\mathrm{CQI}=3$ and $\mathrm{CQI}=7$ because higher modulation scheme (64QAM) is used. It is obvious that, the effect of HARQ retransmissions decreases at higher SNR. This is related to the errors which are decreased significantly at higher SNR.

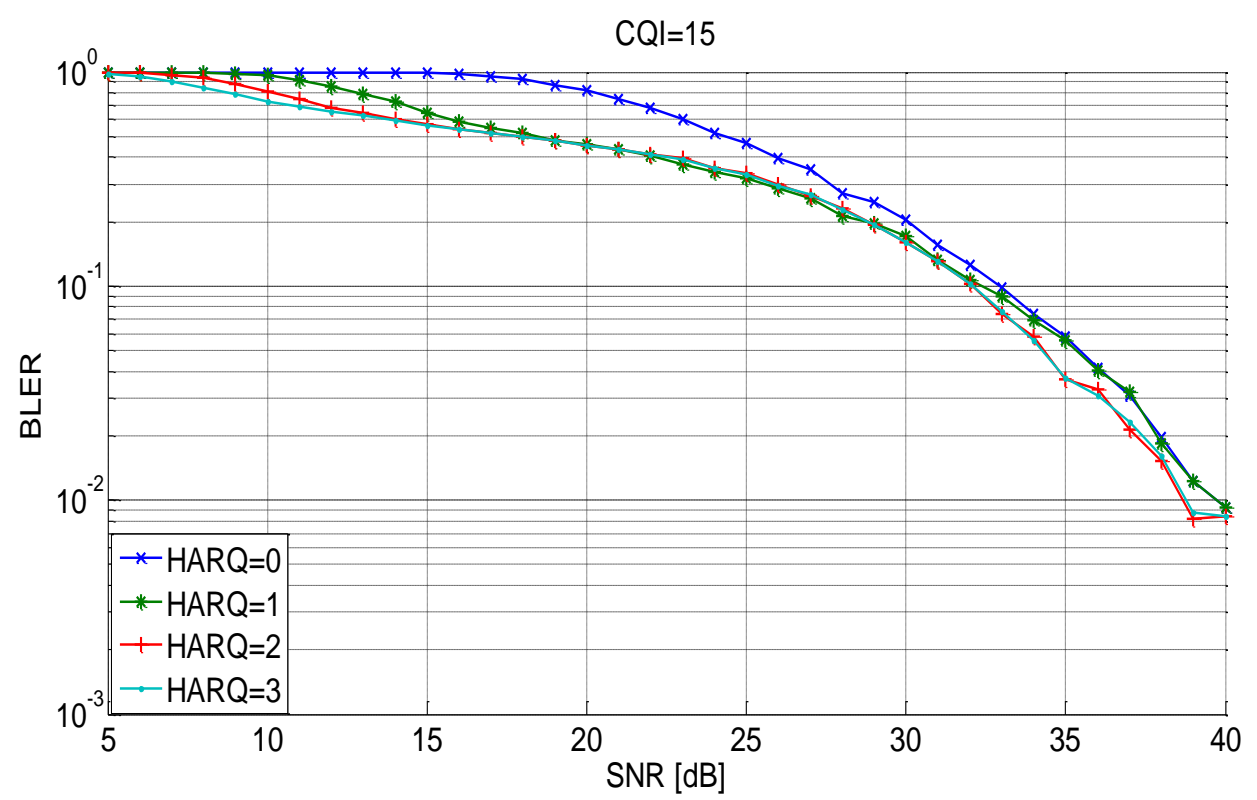

Figure (11): SNR versus BLER when CQI=15. 


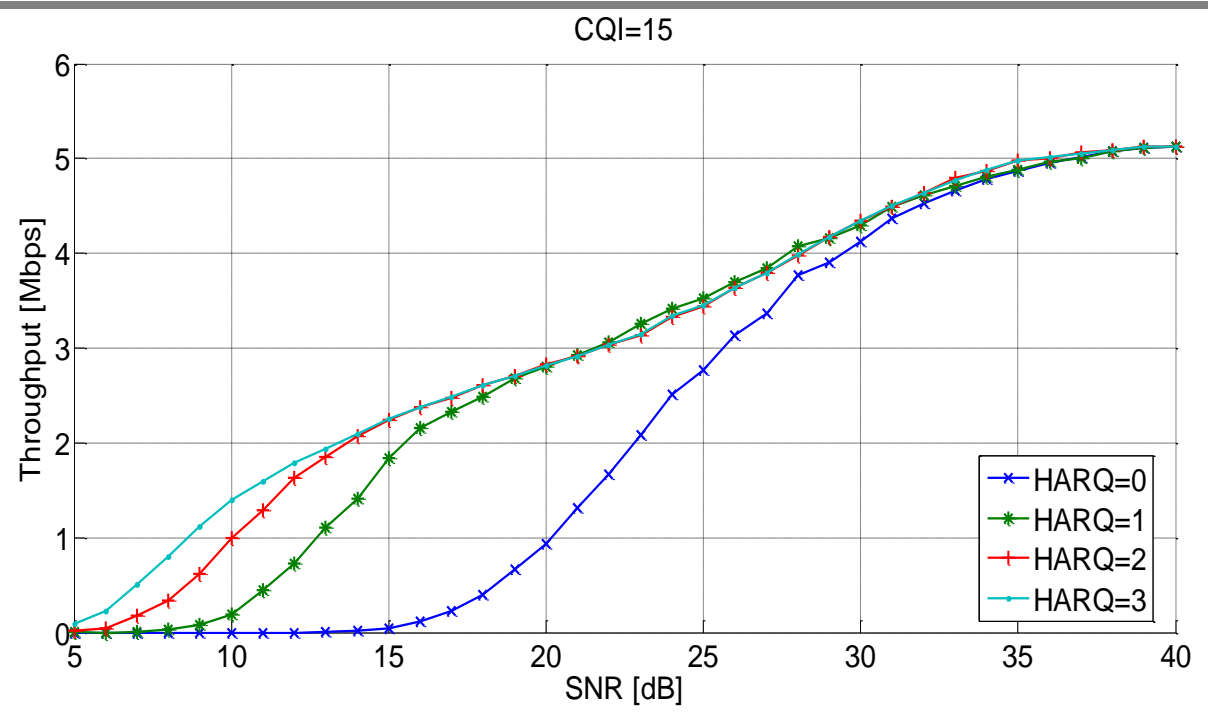

Figure (12): SNR versus Throughput when CQI=15.

Figure (12) illustrates the relationship between SNR and throughput for different HARQ retransmissionsat $\mathrm{CQI}=15$. When HARQprocedure is used the throughput increases twice as compared without using HARQ retransmissionsat SNR equal to $20 \mathrm{~dB}$ then the effect is decreased with the inrement of SNR.

\section{Conclusion}

This paper dealt with the performance of LTE system in the downlink direction and studied the effect of HARQ procedure on the performance of the system. It focused on the BLER and the throughput. The results show that the performance of the system is improved after deploy HARQ procedure from BLER and throughput points of view. It is recommended to deploy one HARQ retransmission for the all cases studied. The number of HARQ retransmissions more than one submits relatively small improvement on the performance of LTE system as well as the processing time which is consumed for retransmissions. It is noted form the results that the effect of HARQ procedure on the performance of the system clearly decreases at the magnitude of SNR higher than $30 \mathrm{~dB}$ due to the errors almost absent.

\section{References:-}

[1] C. Mehlführer, M. Wrulich, J. Colom Ikuno, D. Bosanska, and M. Rupp, "Simulating the Long Term Evolution Physical Layer," in Proc. of the 17th European Signal Processing Conference (EUSIPCO 2009), Glasgow, Scotland, Aug. 2009.

[2] H. Holma and A. Toskala "WCDMA for UMTS: Radio Access for Third Generation Mobile communications", John Wiley \& Sons, Ltd, Fifth Edition, 2010.

[3]K. C.Beh, A.Doufexi and S.Armour, "Performance Evaluation Of Hybrid ARQ Schemes Of 3GPP LTE OFDMA System", The 18th Annual IEEE International Symposium on Personal, Indoor and Mobile Radio Communications, 2007. 
[4]M. T. Kawser, N. I. Bin Hamid, M. N. Hasan, M. S. Alam, and M.M. Rahman "Limiting HARQ Retransmissions in Downlink for Poor Radio Link in LTE", International Journal of Information and Electronics Engineering, Vol. 2, No. 5,September 2012.

[5] Z. He and F. Zhao" Performance Of HARQ With AMC Schemes In LTE Downlink", IEEE, International Conference on Communications and Mobile Computing,2010.

[6] S.Sesia, I.Toufik and M. Baker, "LTE- the UMTS Long Term Evolution, From Theory to Practice", John Wiley \& Sons Ltd., 2011.

[7] J.Zyren, "Long Term Evolution Protocol Overview", White Paper, Freescale Semiconductor, 2008..

[8] J.Zyren, "Overview of the 3GPP Long Term Evolution Physical Layer", White Paper, Freescale Semiconductor, 2007.

[9] 3rd Generation Partnership Project, "Technical Specification Group Radio Access Network; Physical Channels and Modulation (Release -8), 36.211, 2008.

[10] H. Holma and A. Toskala , "LTE for UMTS OFDMA and SC-FDMA Based Radio Access", John Wiley \& Sons, Inc., 2009.

[11] P. Mũnoz, I. de la Bandera, R. Barco, F. Ruiz, M. Toril and S. Luna-Ram'irez " Estimation of Link-Layer Quality Parameters in a System-Level LTE Simulator" ,IEEE, Fifth International Conference on Broadband and Biomedical Communications, 2010. 